Cinémas

Revue d'études cinématographiques

Journal of Film Studies

\title{
Les scénarios de Cap Tourmente
}

\section{Esther Pelletier}

Volume 9, numéro 2-3, printemps 1999

Les Scénarios fictifs

URI : https://id.erudit.org/iderudit/024788ar

DOI : https://doi.org/10.7202/024788ar

Aller au sommaire du numéro

Éditeur(s)

Cinémas

ISSN

1181-6945 (imprimé)

1705-6500 (numérique)

Découvrir la revue

Citer cet article

Pelletier, E. (1999). Les scénarios de Cap Tourmente. Cinémas, 9(2-3), 87-102. https://doi.org/10.7202/024788ar

\section{Résumé de l'article}

Il s'agit d'une étude du processus d'écriture des six versions du scénario du film Cap Tourmente (1993) du scénariste et réalisateur québécois Michel Langlois. Cette analyse prend appui sur une étude à caractère psychanalytique du développement des personnages d'une version à l'autre, de leurs forces d'action, de leur interaction et des événements qu'ils engendrent. L'auteure traque ici le désir qui anime chacun des personnages et tente de décrypter ce qu'ils recèlent et ce qui se cache dans leurs discours. Finalement, elle conclut à une expérience de deuil que vient légitimer le réalisateur du film. 


\title{
Les scénarios de Cap Tourmente
}

\section{Esther Pelletier}

\begin{abstract}
RÉSUMÉ
Il s'agit d'une étude du processus d'écriture des six versions du scénario du film Cap Tourmente (1993) du scénariste et réalisateur québécois Michel Langlois. Cette analyse prend appui sur une étude à caractère psychanalytique du développement des personnages d'une version à l'autre, de leurs forces d'action, de leur interaction et des événements qu'ils engendrent. L'auteure traque ici le désir qui anime chacun des personnages et tente de décrypter ce qu'ils recèlent et ce qui se cache dans leurs discours. Finalement, elle conclut à une expérience de deuil que vient légitimer le réalisateur du film.
\end{abstract}

\section{ABSTRACT}

This article deals with the writing process of the six versions of the scenario for Cap Tourmente (1993) by Quebec scriptwriter and director Michel Langlois. This analysis relies on a psychoanalytic reading of the development of characters from one version to the next (their capacity to act, their interaction and the events they produce). The author traces the desires that set them in motion, attempts to decipher what they conceal and what is embedded in their discourses. As a conclusion, she points to an experience of mourning which, according to her, the director of the film legitimates.

Entre 1993 et 1996, nous avons mené une recherche sur les processus de création dans le cadre d'un projet qui, au départ, s'intéressait au développement d'une ouvre cinématographique'. Généralement, il est difficile d'avoir accès à toutes les 
étapes qui se succèdent lors de l'échafaudage d'un film. Habituellement, le chercheur ne pourra consulter que l'œuvre achevée et, avec un peu de chance, quelques versions du scénario et d'autres documents qui auront pu être déposés dans une institution publique, telle une cinémathèque, ou prêtés parfois par l'auteur ou un producteur.

Toutefois, dans le cadre de notre recherche, nous avions décidé d'étudier un cas type, celui du film Cap Tourmente de Michel Langlois (1993), car nous avions exceptionnellement eu accès à toutes les étapes du processus de création.

En effet, en 1984 et 1985, alors directrice au développement à la $S G C Q^{2}$, nous avons accepté de développer le projet de Michel Langlois, qui s'intitulait à ce moment La Traversée (1984) et qui allait devenir, neuf ans plus tard, Cap Tourmente. Nous avons donc assisté à la naissance du projet cinématographique, dont nous avons pu suivre et lire les premières versions du scénario. Ensuite, au moment d'amorcer notre projet de recherche, Michel Langlois nous donna accès à toute sa documentation, c'est-à-dire à toutes les versions de ses scénarios, à sa correspondance avec les institutions subventionnaires et autres, de même qu'il nous permit d'assister au tournage et au montage du film, en plus de nous accorder des entrevues. Matériel en mains, nous étions prête à nous livrer à notre recherche!

\section{Historique de l'écriture du scénario de Cap Tourmente}

Il y a eu six versions écrites du scénario de Cap Tourmente, étalées sur sept ans de maturation. Même si aujourd'hui, certains producteurs comme Roger Frappier n'hésitent pas à consacrer du temps à l'écriture de plusieurs jets d'un même scénario, en 1984, lorsque Michel Langlois a commencé à concevoir ce qui est devenu Cap Tourmente, c'était tout de même l'exception. Cependant, il faut mentionner que l'écriture de ce film a été, pour le scénariste-réalisateur, le lieu de l'apprentissage de son métier. En effet, au départ, il pensait ne se consacrer qu'à l'écriture, laissant la réalisation à quelqu'un d'autre, au tout début du projet, à la réalisatrice Léa Pool avec qui il avait plusieurs fois travaillé, à titre de coscénariste. Mais la réalisatrice, prise par ses propres projets, fut remplacée par un autre réalisateur, le 


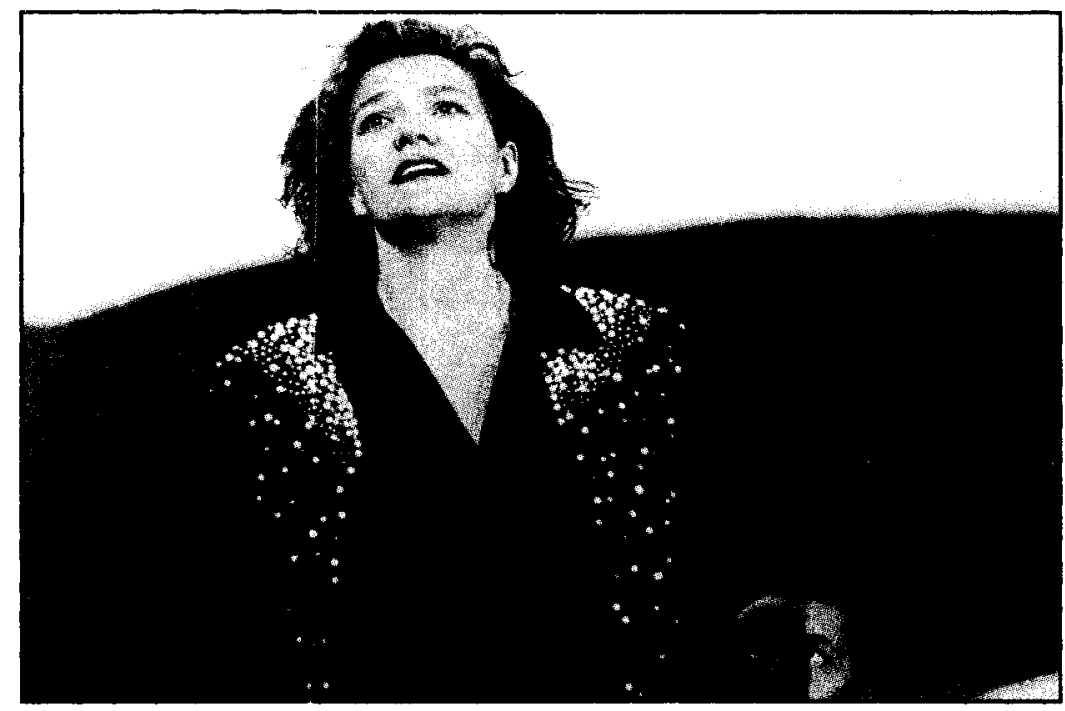

\section{Cap Tourmente de Michel Langlois (1993)}

Français Paul Vecchiali ${ }^{3}$, qui finit lui-même par convaincre Langlois de réaliser son propre scénario. Je cite ce dernier dans une entrevue qu'il nous accordait en janvier 1994 :

$[\ldots]$ je suis passé à la scénarisation parce que je ne me croyais pas équipé pour la réalisation. J'avais peur d'être sur la ligne de front, alors je préférais être dans l'ombre. Mais, à la longue, j'étais malheureux, car pour moi le travail d'écriture est toujours très douloureux. Je voulais faire autre chose et Sortie 234 fut pour moi une réelle surprise. J'étais convaincu que cette expérience me prouverait que je n'étais pas fait pour la réalisation et ce fur le contraire. Sur le plateau, j'étais heureux de toutes les étapes du film. Cap Tourmente m'a mis au monde et non l'inverse.

Finalement, en même temps qu'il écrivait Cap Tourmente, le scénariste dut passer par toutes les étapes habituelles pour devenir réalisateur. Ainsi, en 1988, il réalisa un court métrage, Sortie 234 , ayant pour thème l'identité sexuelle et mettant en vedette pour la première fois à l'écran Roy Dupuis ainsi qu'Élise Guilbeault. Ils allaient devenir, quelques années plus tard, deux des acteurs principaux de Cap Tourmente. Avec ce premier film, 
Michel Langlois remporta le premier prix de court métrage au Festival du cinéma international en Abitibi-Témiscamingue. Puis l'ONF, durant sa période de coproduction avec le secteur indépendant, lui donna l'opportunité de réaliser deux moyens métrages pour la télévision, soit Comme un voleur (1991) et Un même sang (1993), mettant tous deux en scène Gilbert Sicotte ainsi qu'Andrée Lachapelle qui joua le rôle de la mère dans ces deux films. Ces deux comédiens seront également les deux autres principaux interprètes de Cap Tourmente.

\section{Que raconte le film ${ }^{4}$ ?}

Cap Tourmente met en scène les débats affectifs entre une mère (Andrée Lachapelle), son fils (Roy Dupuis), sa fille (Élise Guilbeault) et un ami de la famille, Jean-Louis (incarné par Gilbert Sicotte). Le film débute avec l'arrivée du fils, Alex, coïncidant avec celle de Jean-Louis, dans un petit village de Charlevoix qu'ils ont quitté quelques années auparavant. Ils viennent rejoindre la mère et la sœur d'Alex, soit Jeanne et Alfa, résidant dans une vieille auberge qu'ils ont tous les quatre habitée ensemble neuf ans plus tôt. La diégèse illustre les multiples mouvements du désir qui bouleversent la destinée des quatre protagonistes, les divers affrontements opérant peu à peu une levée du refoulement, mettant à nu leurs motivations et ce qui constituerait, en bonne partie, la cause de leurs désirs. Pourtant ce film pose pour l'analyse un problème majeur: quel est précisément l'objet du désir de chacun des sujets fictifs? Qui désire qui et quelle en est la cause? En effet, le film de Langlois expose une confusion quant à la circulation du désir où quelque chose de la jouissance s'exprime dans un mouvement circulaire, étreignant les personnages principaux dans un étouffement perpétuel. Tourmentés par une douleur innommable, donc indéfinissable, les personnages se heurtent les uns aux autres, tantôt avec tendresse et sensualité, tantôt avec une violence inouie, tant dans l'ordre de la parole que par le biais d'un langage dit sémiotique, notamment celui du corps. De plus, les scènes d'affrontements font sans cesse retour, ordonnées par une compulsion de répétition qui détermine le rapport d'aliénation unissant les personnages. Il nous faut noter toutefois ce paradoxe propre à toute relation objectale: les sujets 
fictifs s'articulent non seulement selon un désir de conjonction d'union -, mais également, et de façon récurrente, selon un désir de disjonction - de désunion. En effet, tous ces personnages se "présentaient" comme des objets aliénés du désir de l'Autre et non comme sujets de leurs désirs. Dans ce "magma" fantasmatique, les figures jouissent jusqu'à l'insupportable, tout en tentant de se dégager de cette jouissance à la fois mortifere et impossible qui les définit, les relie et les confronte en une impasse que seule la parole peut résoudre, en opérant une déliaison nécessaire avec l'Autre, où peut advenir le sujet du désir: en bref, un sujet autonome, mature, s'articulant avec une certaine aisance dans l'ordre du symbolique, dans cette communauté que nous nommons communément la société. Or, voilà ce vers quoi tend justement la diégèse de ce film où la parole se tait, explose, glisse et se reprend afin d'en arriver à l'expression d'un désir secret, enfoui, qui caractériserait chacun des sujets fictifs ici concernés, par le biais de la reconnaissance par la parole pleine d'un désir propre à chacun, les distinguant et les libérant de l'emprise totalisante du désir de l'Autre. C'est cette traversée fictive et langagière du fantasme, comme encadrement du désir, qui permet la coupure nécessaire à l'individuation du sujet. D'ailleurs, le scénario de Langlois s'intitulait à l'origine La Traversée. Il s'agissait alors des quatre premières versions (mars, avril et septembre 1985, puis juillet 1989). Quelques années plus tard, l'auteur réintitula son scénario Ressac, nom commun désignant le retour violent des vagues sur ellesmêmes, lorsqu'elles se brisent contre un obstacle. Ces deux titres, non retenus pour la version finale, sont dignes d'intérêt en ce qu'ils démontrent tout à fait les mouvements du désir, ce deus ex machina provoquant la production de la diégèse, soit le "retour " violent d'un désir incessant et le repliement narcissique sur soimême après la rencontre souvent brutale de l'"obstacle" quant à la pleine satisfaction de ce désir, ainsi que la "traversée" de ce long voyage, que pourrait illustrer la cure analytique, dans lequel le sujet élabore en signifiants son désir pour en déterminer la cause. Ressac et traversée, ces deux métaphores paradoxales l'une par rapport à l'autre définissent les voies mystérieuses qu'empruntent le désir. Cap Tourmente de Michel Langlois illustre avec une poésie conforme à l'ensemble de son œuvre cinématographique les 


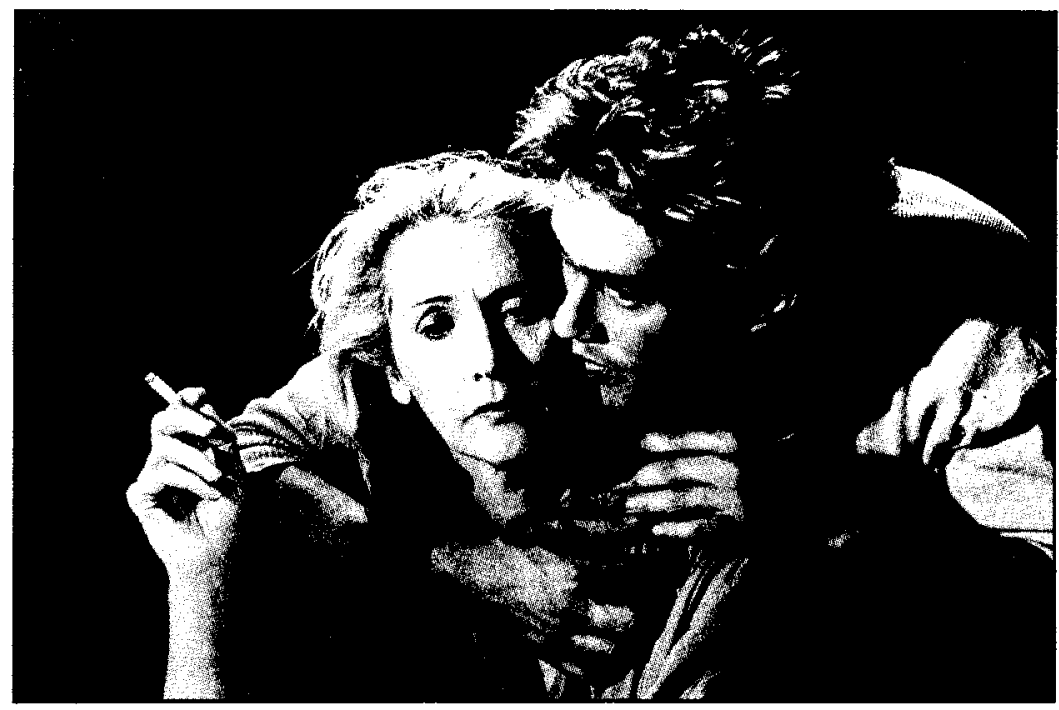

\section{Cap Tourmenfe de Michel Langlois (1993)}

profondeurs et les orientations de désirs les plus souvent individuellement et socialement refoulées, exposées ici à travers une écriture et des représentations singulières, propres à l'imaginaire de l'auteur.

\section{Les différentes versions: personnages et situations narratives}

Nous rendrons compte ici des modifications majeures qu'a subies le récit original, à travers la rédaction des différentes versions, avant d'aboutir à l'œuvre finale. Nous parlerons uniquement des personnages et de leurs motivations, ainsi que des situations narratives dans lesquelles ils s'incarnent.

Après analyse, nous avons constaté qu'il y a deux moments marquants qui se dégagent du processus d'écriture élaboré par l'auteur. D'abord, un premier moment où furent écrites les versions de mars, d'avril et de septembre 1985 en collaboration avec le réalisateur Paul Vecchiali. Ces versions se ressemblent; elles présentent la même problématique familiale. Elles furent écrites dans un laps de temps assez rapproché, soit sept mois. On remarque, d'une version à l'autre, que les motivations profondes des personnages demeurent les mêmes, mais que leurs caractéristiques se précisent. 
Ensuite, quatre années s'écoulent avant que Langlois reprenne l'écriture de Cap Tourmente qu'il avait délaissée, Vecchiali s'étant entre-temps désisté et Langlois s'étant consacré à la réalisation de deux films. Ce n'est qu'en 1989 qu'il se remet donc à l'écriture, mais cette fois en collaboration avec le scénariste Marcel Beaulieu 5 .

Ce second temps de l'écriture relance le scénario sur une autre piste, transformant la problématique de départ. C'est qu'entre-temps, l'auteur s'est affirmé et, comme nous le verrons, ce changement s'inscrit dans l'élaboration de ses personnages.

Voici les tableaux schématiques qui illustrent ces modifications, de même que leurs analyses commentées.

\section{TABLEAU 1}

\section{VERSIONS}

Mars 1998 La Traversée

de Michel Langlois

et de Paul Vecchiali

Avril 1985

Septembre 1985

MOTIVATIONS - DÉSIRS DES PERSONNAGES

PÈRE - Marin

- Décédé

- Aimé de Jeanne

JEANNE Mère (68 ans)

- Veuve

(Andrée Lachapelle)

- Attend le retour de son fils Alex

Prétexte: rouvrir son auberge

JULIEN Ami de la famille (45 ans) (Gilbert Sicotte)

- Fuit son existence ne sachant pas très bien comment l'orienter

Prétexte : participer à la réouverture de l'auberge où il a déjà travaillé.

ALEX Fils de Jeanne (32 ans) (Roy Dupuis)

- Marin

- Attaché à sa mère, ne peut et ne veut pas prendre sa vie en main

- Recherche d'un bonheur inaccessible 
- Provoque Julien

Prétexte: prendre en main l'auberge de sa mère

BARBARA Amante d'Alex (25-28 ans)

(Macha Limonchik)

- Amoureuse d'Alex

- A travaillé avec lui sur un bateau

- Américaine

M. LÉON Villageois (65 ans)

(Gabriel Gascon)

- Chauffeur de taxi

- Désire un lien amoureux avec Jeanne

$\begin{array}{ll}\text { ALFA Employée de l'auberge (40 ans) } & \text { (Élise Guilbault) } \\ & \text { A eu une relation intime avec Alex } \\ & \text { Toujours amoureuse de lui }\end{array}$

FINALE: Fatiguée d'attendre Alex qui repart naviguer, Jeanne propose à Julien de reprendre l'auberge

Dans le tableau 1, établi à partir des premières versions du scénario, le père, marin de profession, est décédé. Selon sa femme, Jeanne, 68 ans, il l'a aimée. La diégèse débute alors que Jeanne est veuve et attend le retour de son fils Alex pour rouvrir l'auberge qu'elle a déjà gérée quelques années auparavant. Surgit Julien, la quarantaine, ami de la famille et ancien employé de l'auberge. Il désire opérer un changement dans son existence et participer à la réouverture de l'auberge semble le prétexte indiqué. Alex, la trentaine, est l'autre personnage pivot de la diégèse. Tout comme son père, il est marin. Cependant, il n'est pas heureux de sa situation, préférant se réfugier auprès de sa mère avec le prétexte lui aussi de reprendre l'auberge en main avec elle. Mais Alex n'est jamais heureux là où il est. Il veut toujours être ailleurs. Débarque alors parmi eux Barbara, amoureuse d'Alex. Elle a travaillé avec lui sur un bateau. S'ajoutent deux personnages secondaires: M. Léon, 65 ans, chauffeur de taxi de la région, amoureux de Jeanne, ainsi qu'Alfa, 40 ans, employée de l'auberge qui a déjà eu une relation amoureuse avec Alex et qui l'aime toujours.

Avec de telles motivations habitant les personnages, tout est en place dans ces versions pour explorer le désir de chacun et leur donner le potentiel d'interagir entre eux. Après de multiples méandres situationnels où s'expriment leurs désirs, la diégèse se 
clôt de la façon suivante: fatiguée d'attendre Alex qui repart naviguer, Jeanne propose à Julien de reprendre l'auberge.

\section{TABLEAU 2}

\section{VERSION}

Juillet 1989 La Traversée

de Michel Langlois

en collaboration

avec Marcel Beaulieu

\section{MOTIVATIONS - DÉSIRS DES PERSONNAGES}

\section{PÈRE}

- Marin

- Décédé

- Aimé de Jeanne

JEANNE Mère (55- 60 ans)

- Vit avec Alex son fils

- Craint la violence d'Alex

- Aime Scott en secret, désire qu'il remplace Alex

- Elle téléphone à Scott (l'ancien Julien)

Prétexte: besoin d'aide pour gérer l'auberge

SCOTT Ami de la famille (45 ans)

- Revierit par nostalgie

- A déjà travaillé à l'auberge

- Amoureux d'Alex

Prérexte: revenir travailler à l'auberge

ALEX Fils de Jeanne (26-27 ans)

- Instable

- Ne trouve pas de travail

- A joue du violoncelle

- Ne parvient pas à se séparer de sa mère

- Amoureux de sa soeur Alfa

- S'amuse avec les sentiments de Scott

ALFA Fille de Jeanne (30 ans)

- Est devenue la fille de Jeanne et la sceur d'Alex

- Réside au village

- Travaille dans un bar

- Éprouve une artirance envers Scott

- Entretient une relation tendue avec sa mère

- A plusieurs aventures sentimentales 
- Aime son frère Alex

- Vient travailler à l'auberge

BARBARA Amante d'Alex (20 - 22 ans)

- Touriste itinérante

- Aboutit à l'auberge pour faire réparer sa bicyclette

- Alex tombe amoureux d'elle

- Se fait engager à l'auberge pour faire le ménage

M. LEON Villageois (65 ans)

- Chauffeur de taxi

- Désire un lien amoureux avec Jeanne

FINALE: Barbara, Alfa et Alex s'en vont. Jeanne reste seule avec Scott, ce qu'elle désire

Le tableau 2 illustre les motivations des personnages, ceux de la version de 1989 , toujours intitulée La Traversée. Cette version est le fruit du premier travail de collaboration entre Michel Langlois et le scénariste Marcel Beaulieu. Dans cette version, les personnages se précisent davantage à l'exception du père et de M. Léon dont le profil et les motivations demeurent semblables. Jeanne, quant à elle, est plus jeune (55-60 ans), elle vit avec Alex, son fils, dont elle craint les accès de violence. Elle aime secrètement Scott - Julien dans les versions précédentes - et fait appel à lui pour gérer l'auberge afin de remplacer Alex. Quant à Scott, qui a travaillé autrefois dans cette auberge, il est amoureux d'Alex et il revient par nostalgie. Dans cette version, Alex est dans la vingtaine (26-27 ans). Il est instable, n'arrive pas à quitter sa mère ni l'auberge et est amoureux de sa sœur Alfa (simple employée de l'auberge dans les autres versions). De plus, il joue avec les sentiments de Scott. De plus, les auteurs lui ont ajouté une activité supplémentaire, qui apparaîtra plus tard à l'écran, celle de jouer du violoncelle.

Quant à Alfa, son rôle a acquis de l'importance dans cette version; son statut a changé. D'employée de l'auberge, elle est devenue membre à part entière de la famille. Elle a 30 ans, elle vit au village, travaille dans un bar, entretient une relation tendue avec sa mère, a plusieurs aventures sentimentales, aime son frère Alex et décide également de travailler à l'auberge. Barbara, elle, devient une touriste allemande qui aboutit à l'auberge pour 
faire réparer sa bicyclette. Elle tombe amoureuse d'Alex et se fait engager à l'auberge pour faire du ménage.

A la fin du parcours narratif des personnages, Barbara, Alfa et Alex s'en vont, laissant Jeanne seule avec Scott, ce qu'elle désire.

\section{TABLEAU 3}

\section{VERSION}

Mai 1991, Mars 1992 Ressac

de Michel Langlois

en collaboration avec

Marcel Beaulieu

\section{MOTIVATIONS - DÉSIRS DES PERSONNAGES}

PÈRE

- Marin

- Décédé

- Aimé de Jeanne

JEANNE Mère (55-60 ans)

- Aime Jean-Louis (ex. : Julien - Scott)

- Voudrait ressusciter le bonheur passé lorsque l'auberge fonctionnait bien

- Blessée par la mort de son mari

- Souhaite qu'Alex prenne son envol

- Alex lui rappelle son défunt mari

- Jean-Louis lui réapprend à conduire sa voiture

Prétexte: Besoin d'aide pour gérer l'auberge

JEAN-LOUIS (Julien-Scott) Ami de la famille O'Neil (45 ans)

- Ancien employé de l'auberge

- Il se sent seul après le décès de sa propre mère

- Revient par nostalgie

Mai 1991 - Homosexuel

- Aime Alex

- Ami de la famille O'Neil

- Ancien employé de l'auberge

Mars 1992 - Rival d'Alex auprès de Jeanne et d'Alfa

- Il aime Jeanne

- Il fait office de mari, père, frère, fils et amant

ALEX Fils de Jeanne (26-27 ans)

- Marin à ses heures

- Est traqué; a besoin d'argent 
Mai 1991 - Perturbé; instable

- Jean-Louis lui propose de partir avec lui

Mars 1992 - Force Alex à prendre sa vie en main

ALFA Fille de Jeanne; soeur d'Alex (30 ans)

- Elle veut devenir propriétaire d'un restaurant

- Aime Alex et le protège

- A déjà aimé Jean-Louis et l'aime toujours

BARBARA Touriste itinérante (20 - 22 ans) (25 ans)

- Amoureuse d'Alex

- Aime la famille O'Neil

- Déçue par Alex, elle repart

M. SIMON (Ex.-M. Léon Villageois (65 ans))

- Client de l'auberge depuis une quinzaine d'années

- Veuf

- Veut épouser Jeanne

WILFRED Amoureux d'Alfa (35 ans)

- L'épouse

FINALE: Jeanne vend son auberge à Alfa qui se marie avec Wilfred. Barbara part et Jean-Louis force Alex à prendre sa vie en main avant de partir lui aussi. Jeanne est prête à partir à son tour

Le tableau 3 illustre les deux dernières versions du scénario avant le tournage. Elles sont assez fidèles à l'œuvre cinématographique. À la lecture de ces deux scénarios, on constate que seul le personnage du père n'a pas changé. Il est identique depuis le début du projet, et il le restera dans la diégèse du film. Quant à Jeanne, tout comme dans le film, elle aime Jean-Louis (Julien et Scott dans les versions antérieures) et voudrait revivre le passé, lorsque l'auberge fonctionnait bien. Elle souffre de la mort de son mari, qu'Alex, son fils, lui rappelle, mais elle parvient cependant à désirer que celui-ci prenne son envol et quitte la maison familiale. Jean-Louis, pour sa part, est toujours un ancien employé de l'auberge qui revient, éprouvant un sentiment de nostalgie. Cependant, entre la version de 1991 et celle de 1992 s'opère un important changement. En effet, d'homosexuel amoureux d'Alex et se sentant seul après le décès de sa propre mère (version de 1991), Jean-Louis devient, dans la version de 1992, le rival d'Alex auprès de Jeanne et d'Alfa, et il aime 
Jeanne. Dans cette famille, il occupe la fonction symbolique d'époux, de père, de frère, de fils et d'amant. Cette dernière version est la plus fidèle à l'œuvre cinématographique, à ceci près que tout est nuancé dans le film: le désir de Jean-Louis et des autres personnages est la plupart du temps suggéré, refoulé, puis parfois dévoilé.

Alex, quant à lui, est marin à ses heures. Dans ces versions, tout comme dans le film, il est pourchassé et a besoin d'argent. On en ignore cependant la cause exacte, probablement une affaire de drogue; cela n'est pas clairement précisé. Il est évident maintenant qu'il s'agit d'un être perturbé et instable. Toutefois, un changement important s'est opéré entre la version de 1991 et celle de 1992. En 1991, l'interaction entre Alex et Jean-Louis se clôt par la proposition de Jean-Louis faite à Alex de partir avec lui; tandis que dans la version de 1992, qui est fidèle au film, Jean-Louis force Alex à quitter la maison familiale. Il le conduit à la croisée des chemins et l'exhorte à "aller dans sa vie", tout comme le ferait un père.

Le personnage d'Alfa, la fille et la sœur de la famille, se précise et se complexifie dans ces versions et dans le film. Elle veut un restaurant qui lui appartienne. Elle aime Alex et le protège démesurément. Elle a déjà aimé Jean-Louis et l'aime encore. Dans le film, on assiste à une réelle évolution du personnage. Tout comme les autres, elle est amenée à préciser son désir et à l'affirmer à la fin de la diégèse, après plusieurs hésitations. C'est ainsi qu'elle épousera Wilfred, un ami de cœur, et avec ses économies, qu'elle achèrera l'auberge familiale pour la gérer avec lui.

Barbara est cette autre femme que le charme d'Alex ne laisse pas indifférente. Touriste allemande débarquée par hasard à l'auberge, elle tombe amoureuse d'Alex qui, dans un premier temps, ne la repousse pas. Elle aime la famille O'Neil; mais déçue du comportement d'Alex qui devient violent et indifférent, elle quitte l'auberge.

Dans ces versions et dans le film, le personnage de M. Simon, l'ex-M. Léon, le chauffeur de taxi, est devenu un client assidu de l'auberge depuis une quinzaine d'années. Veuf et amoureux de Jeanne, il veut l'épouser. Mais Jeanne préfere demeurer fidèle à son défunt mari et... à ses enfants. 
Nouveau personnage apparaissant dans ces versions et dans le film, Wilfred est l'ami de cour l'Alfa. Amoureux, il finit par l'épouser.

La fin du parcours narratif de ces versions et du film se clôt par la vente de l'auberge de Jeanne à Alfa qui épouse Wilfred, le départ de Barbara et celui forcé d'Alex provoqué par Jean-Louis, avant que lui-même ne quitte. Quant à Jeanne, à la toute fin, elle serait prête à partir à son tour de son côté.

Par ailleurs, nous nous pencherons maintenant sur un "personnage-lieu» qui nous semble important et significatif dans la diégèse, soit l'auberge. Les transformations et le rôle que joue l'auberge comme matrice, lieu de rassemblement, sorte d'utérus, site matriarcal qui regroupe la famille, sont également porteurs de sens.

Dans les versions de 1985, l'auberge est fermée. Pour Jeanne, il s'agit de la rouvrir avec Alex. Mais, à la toute fin, comme ce dernier s'avère incompétent, c'est avec Julien que Jeanne veut réaliser son projet. Dans la version de 1989, l'auberge vit encore, mais Jeanne a besoin d'aide pour la faire bien fonctionner. Elle fait appel à Scott pour l'aider, Alex s'avérant violent et incompétent.

À la toute fin, Alex, Alfa et Barbara s'en vont, laissant Jeanne et Scott ensemble à l'auberge, formant comme un nouveau couple.

Enfin, dans les deux dernières versions et dans le film, l'auberge bat de l'aile et Alex de même que Jean-Louis et Alfa veulent bien mettre la main à la pâte pour la sauver. Cependant, à mesure que les désirs de chacun se précisent de même que les conflits, le projet de l'auberge en subit les conséquences. Ainsi, à la toute fin, Jeanne vend son auberge à Alfa et chacun part de son côté, suivant en cela leur propre destinée.

Ainsi, l'auberge est ce lieu symbolique, substitut de la mère, qui sert de prétexte à attirer la famille en son sein de manière à tenter de la garder soudée à jamais alors que la socialisation de chaque individu et la prise en charge de l'autonomie de chacun commande son démantèlement. Il aura fallu six versions du scénario et neuf ans d'écriture afin de parvenit à cette conclusion. 


\section{Analyse du parcours du désir des personnages}

\section{d'une version à l'autre}

Dans les premières versions (1-2-3-4), où les personnages se complexifient d'une version à l'autre, on assiste donc à l'histoire "d'une mère qui ne veut pas se détacher de son fils, Alex». Ce dernier ressemble physiquement à son mari décédé des années plus tôt.. Après avoir espéré le retenir à la maison familiale en lui proposant de reprendre l'auberge, Jeanne, qui en est la propriétaire, doit se résigner à ne pas compter sur son fils. Aussi son désir se déplace-t-il vers Julien-Scott, nouvellement débarqué dans cet univers trouble. Julien-Scott est en crise et ne sait où se réfugier. Le projet de l'auberge peut être une solution pour lui. Cependant, Julien-Scott désire Alex. Puis surgit Barbara, l'étrangère, l'Américaine, qui elle aussi est amoureuse d'Alex. Ils ont travaillé ensemble sur un bateau. Au cours de ces versions s'ajoute un autre personnage, plutôt effacé, celui d'Alfa. Ce personnage travaille à l'auberge comme femme de ménage et désire Alex depuis plusieurs années.

\section{SCHÉMA 1}

\section{L'orientation du désir}

dans les quatre premières versions du scénario de Cap Tourmente (1993) de Michel Langlois

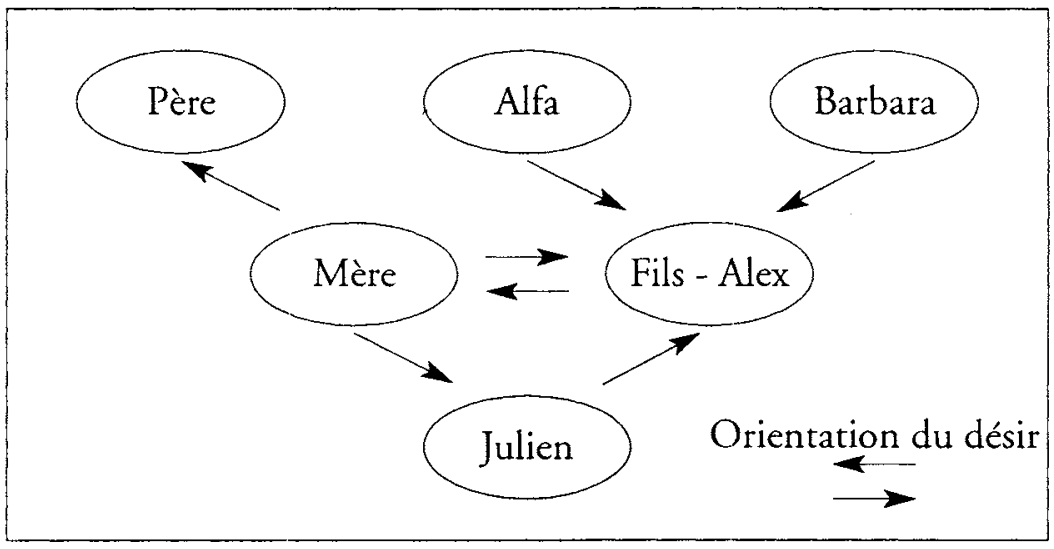

Finalement, dans les deux dernières versions - dont la version finale avant le tournage du film -, la situation s'est 
inversée. Il y a eu «ressac». En effet, on assiste alors à l'histoire "d'un fils, Alex, qui ne veut pas se détacher de sa mère", mère qui l'a toujours chéri, d'autant plus qu'il ressemble à son mari décédé quelques années plus tôt.

Alex est également chéri par sa sœur Alfa, qui le protège et qui s'oppose à sa mère. Alfa, bien que travaillant dans un bar du coin, n’a pas non plus réussi à quitter le toit familial. Elle a cependant un nouvel ami de coeur, Wilfred, qui en est très amoureux. Dans cette version, Alfa est devenue un personnage central. Débarque également dans cet univers, Jean-Louis, ami de la famille, financièrement à l'aise, libre comme l'air, ayant tout quitté pour recommencer une nouvelle vie. Puis surgit Barbara, la touriste allemande égarée qui se réfugie à l'auberge et qui tombe amoureuse d'Alex.

Avec l'arrivée et l'aide de Jean-Louis, chaque membre de la famille, mère, fils, fille, frère, sœur, réussira à se séparer pour aller vers sa destinée. Jean-Louis jouera symboliquement le rôle du père absent en séparant la mère de ses enfants permettant ainsi la socialisation de ceux-ci. Cependant, cette séparation ne se fera pas sans heurts, chacun devant renoncer à sa dépendance pour en faire le deuil.

\section{SCHÉMA 2}

\section{L'orientation du désir}

dans les cinquième et sixième versions du scénario de Cap Tourmente (1993) de Michel Langlois

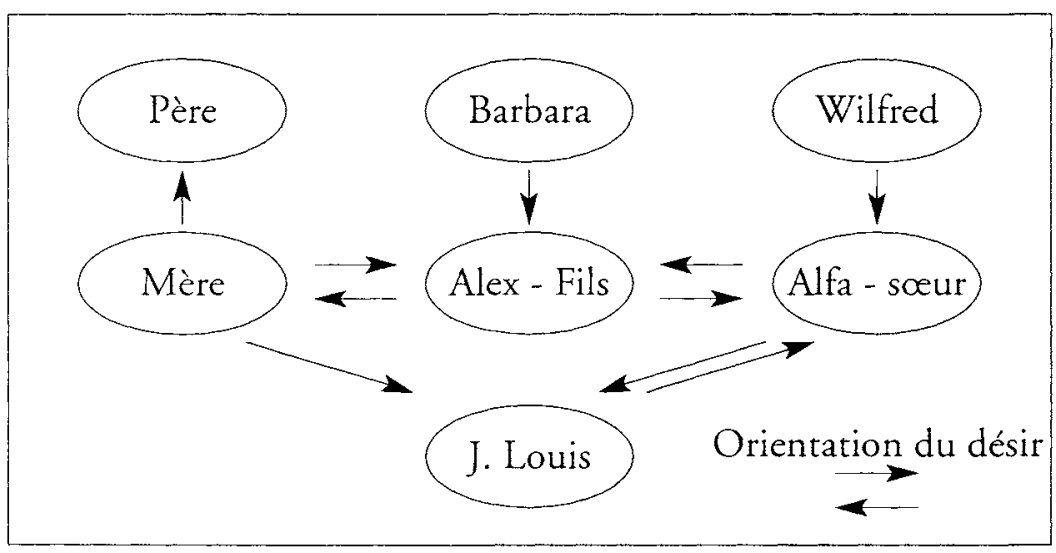


À y regarder de plus près, La Traversée met d'abord en scène l'échafaudage d'un rapport incestueux et le désir, en partie inconscient, de le concrétiser. Au fil de l'écriture des différentes versions, le désir de l'auteur se modifie ${ }^{6}$. En effet, dans Ressac, le scénario, et Cap Tourmente, le film, Langlois donne à ses personnages des caractéristiques et des moyens pour rompre cette structure. Alfa a un travail et part avec son "chum» qui l'aime; Barbara quitte Alex, Jean-Louis est à l'aise financièrement et autonome; Jeanne, la mère, veut reprendre le commerce de son auberge. Bref, tout le contraire des premiers jets. $\grave{A}$ notre avis, un long travail de deuil de la part de l'auteur s'est réalisé au cours de l'écriture du film. Et ce deuil se concrétise en quelque sorte à travers ses personnages au moment où il les quitte à la fin de Cap Tourmente. En effet, la mère doit faire le deuil de son fils, le fils ainsi que la fille doivent faire celui de leur mère et Jean-Louis doit faire le deuil d'Alfa et de cette famille qui pour lui fait figure de substitut maternel. Barbara renonce aussi à Alex et Alfa se sépare de son frère.

Lhistoire de l'écriture des scénarios de Cap Tourmente, après analyse, est donc l'histoire de "la traversée» d'une structure incestueuse où il y a eu "ressac", ce qui a permis aux personnages tourmentés par leur désir et celui de l'Autre de se séparer. Nous ajouterions également que Cap Tourmente est l'«adaptation" d'un travail de deuil de l'auteur en une création; création qui lui a permis de s'adapter à la vie en se vouant à un travail de deuil via l'écriture d'un scénario et la réalisation de ce film.

En conclusion, nous citerons Michel Langlois dans une lettre qu'il adressait au chercheur Steven Morin, qui à notre suggestion lui a fait parvenir les résultats de nos recherches:

Quant à votre texte "Les tourments du désir maternel ", je le trouve passionnant. La notion de deuil entre autres était très importante pour moi. Deuil du père, deuil du mari, deuil de la loi ancienne, etc. Mais jusqu’à présent, personne ne l'avait soulevée. Du seul fait de vous lire, je me sens tout à coup un peu moins seul dans cette aventure de Cap Tourmente qui aura été la plupart du temps si solitaire. Merci. Michel Langlois.

Université Laval 


\section{NOTES}

1 Il s'agissait du projet Cap Tourmente, processus de création: de lidée au film, subvention du FCAR, 1993-1996.

2 Société générale du cinéma du Québec, devenue en 1994 la SOGIC, Société générale des industries culturelles du Québec.

3 Réalisateur, entre autres, de Les Ruses du diable (1965), Femmes, films (1974), La Machine (1977), Corps à coeur (1979), En haut des marches (1985).

4 Pour cette partie, nous empruntons au travail de recherche de l'assistantchercheur de 3" cycle, M. Steven Morin, qui travailla sur le projet en 1995 et 1996.

5 Marcel Beaulieu est le coscénariste, entre autres, de Farinelli (1994) du réalisateur belge Gérard Corbiau.

6 En ce qui a trait aux "projections" de l'auteur à travers son œuvre, voir un autre travail à paraître que nous avons consacré à cette question, L'adaptation dans tous ses états (Québec: Nota Bene, 1999), notamment le chapitre "Adaptation et création". 